\title{
Bismuth-Coated Mesoporous Platinum Microelectrodes as Sensors for Formic Acid Detection
}

\author{
Salvatore Daniele,* Carlo Bragato, Dario Battistel \\ Dipartimento di Scienze Molecolari e Nanosistemi, Università Cà Foscari Venezia, Calle Larga, S. Marta, 2137, 30123 Venice, Italy \\ tel: +39-041-2348630; fax: + 39-041-2348594 \\ *e-mail: sig@unive.it
}

Received: September 29, 2011

Accepted: November 4, 2011

\begin{abstract}
Mesoporous platinum microeletrodes (MPtEs) modified by sub-monolayers of irreversibly adsorbed bismuth (BiMPtE) were investigated for their potential use as sensors for the detection of formic acid in direct formic acid fuel cells. The mesoporous platinum films were prepared by electrodeposition of platinum on Pt microdisks substrates $25 \mu \mathrm{m}$ diameter, from hexachloroplatinic acid dissolved in the aqueous domain of the lyotropic liquid crystalline phase of octaethylene glycol monohexadecyl ether. The roughness factor $(R F)$ of the MPtEs was about two orders of magnitude greater than those of the corresponding polished microelectrodes. Bismuth ad-atoms onto the platinum surface were deposited by under potential deposition from $1 \mathrm{mM} \mathrm{Bi}^{3+}$ ions in $0.5 \mathrm{M} \mathrm{H}_{2} \mathrm{SO}_{4}$ solutions. The catalytic activity of a series of Bi-MPtEs, characterized by different roughness and fractional bismuth coverage $\left(\theta_{\mathrm{Bi}}\right)$, towards the oxidation of $\mathrm{HCOOH}$, was investigated by cyclic voltammetry and potential step experiments. Compared to MPtEs, Bi-MPtEs displayed enhanced electrooxidation currents at lower potentials. The stability of irreversibly adsorbed bismuth, and consequently the Bi-MPtEs catalytic activity, was found to depend on the high potential limit employed in the measurements. In general, both electrode stability and electrocatalytic performance were good, provided that the operational potential was kept $\leq 0.4 \mathrm{Vvs}$. Ag/AgCl. Bi-MPtEs with $\theta_{\mathrm{Bi}}>0.3$ provided almost sigmoidal shaped waves with low hysteresis, as those expected for microelectrodes working under steady state. The effect of concentration of $\mathrm{HCOOH}$ was investigated over the range $0.01-5 \mathrm{M}$, and linearity between current and concentration depended on both roughness factor and bismuth coverage. A Bi-MPtE characterised by $R F=210$ and $\theta_{\mathrm{Bi}} \geq 0.6$ provided linearity up to $2 \mathrm{M}$ of formic acid. Reproducibility of the sensors was within $2 \%$ $(R S D)$. The same sensor, under the optimized experimental conditions, could be employed for at least two months with negligible loss of the initial performance.
\end{abstract}

Keywords: Formic acid electrooxidation, Mesoporous platinum, Bismuth-coated microelectrodes, Bismuth-UPD, Formic acid sensor

DOI: 10.1002/elan.201100549

\section{Introduction}

Fuel cells powered by small organic molecules (SOMs) such as methanol, ethanol and formic acid have rapidly progressed due to rising energy demands, depletion of fuel reserves and environmental pollution issues [1-6]. Polymer electrolyte membrane fuel cells based on SOMs are promising, in particular, for application in portable electronic devices $[1,2]$. Among SOMs, formic acid has gained special interest as a fuel for direct formic acid fuels cells (DFAFC), because of good power densities, high performance at relatively low temperature [7], and lower crossover rate through the Nafion membranes compared with methanol [2-6]. The latter phenomenon is a significant issue limiting the cell performance of a fuel cell based on SOMs. It, in fact, reduces fuel utilization, results in a detrimental mixed potential, and SOMs can act as poisons for the cathode material. Therefore, SOMs sensors are required to detect their level both in the fuel fed in the anodic compartment of the cell and in the cathodic zone where SOMs can permeate through the membrane.

Electrochemical sensors are suitable to this purpose, as they allow easy and rapid detection of compounds even on line with simple and relatively inexpensive apparatuses [8]. Electrochemical sensors for SOMs, especially for methanol, have been developed and they are basically arranged as small fuel cells [9]. Although, under given conditions, these sensors display satisfactory performance, their operation suffers from the need of external feeding of oxygen or air to the cathode to allow a complete consumption of the fuel that diffuses to the anode/membrane surface.

In this paper we investigate the performance of single miniature electrodes that can operate over a formic acid concentration range, which can be expected to diffuse to the cathode zone of a DFAFC. The sensors here developed are based on microelectrodes (i.e., electrodes with a critical dimension $\leq 50 \mu \mathrm{m}$ ) [10], which are coated by ma- 
terials that are employed as catalysts for the efficient electrooxidation of formic acid.

As it is known, platinum-based catalysts are among the most efficient materials for formic acid oxidation [11,12]. However, platinum is susceptible to poisons such as $\mathrm{CO}$, which strongly adsorbs onto the electrode surface active sites resulting in a dramatic decrease in catalytic efficiency $[3,4,13]$. Under given conditions, the activity of platinum towards the electrooxidation of $\mathrm{HCOOH}$ can be promoted by either using nanostructured platinum electrodes [13-15] or platinum electrodes modified by foreign metal ad-atoms [16-24].

Platinum electrodes with high surface areas and welldefined periodic nanostructures have recently received increasing interest, because of their potential applications in electrocatalysis and electroanalysis $[14,16,19,20,21,25-$ 31]. These electrode systems can be prepared by templating techniques from lyotropic liquid crystalline phases of non-ionic surfactants [32-35]. In particular, the normal topology hexagonal $\left(\mathrm{H}_{\mathrm{I}}\right)$ liquid crystalline phase has been used as a template for the synthesis of mesoporous platinum thin films via the electrochemical reduction of platinum salts dissolved in the aqueous domain of the liquid crystalline phases [32-35]. The pore diameters of the $\mathrm{H}_{\mathrm{I}^{-}}$ $\mathrm{Pt}$ films reflect those of the liquid crystal structure and typically range between 2 and $5 \mathrm{~nm}$ [32-35]. Depending on the thickness of the Pt films, which can be controlled by the deposition charge, the real surface areas of the electrodes can be up to two or three orders of magnitude greater than those of the bare electrodes.

Mesoporous platinum films have been employed for the electrooxidation of methanol and formic acid, and because of their high surface areas, they have displayed poisons-tolerance behaviour $[13,25,26]$. Moreover, modification of the Pt surface with ruthenium has provided further improvements of the electrode activity towards formic acid electrooxidation [21]. In a preliminary work, $\mathrm{H}_{\mathrm{I}}-\mathrm{Pt}$ microelectrodes modified by sub-monolayers of bismuth (Bi-MPtEs) have also been employed for the electrooxidation of $\mathrm{HCOOH}$ [20]. However, the activity of these types of electrodes can critically be affected by the real surface areas, bismuth coverage and stability of the latter onto the electrode surface. These aspects are investigated in detail here to assess the suitability of a series of MPtEs and Bi-MPtEs to be employed as sensors for monitoring $\mathrm{HCOOH}$ in DFAFC. The mesoporous platinum microelectrodes can be advantageous, as they combine the high real surface area of the nanostructure with efficient mass transport characteristics [10]. Moreover, they allow measurements to be performed at high current densities with negligible effects due to voltage drop [10] even in fuels that are characterized by relatively low ionic strength [36,37].

\section{Experimental}

\subsection{Chemicals and Reagents}

All chemicals employed were of analytical reagent grade. Hexachloroplatinic acid (HCPA), potassium chloride, the surfactant octaethylene glycol monohexadecyl ether $\left(\mathrm{C}_{16} \mathrm{EO}_{8}\right), \mathrm{Bi}\left(\mathrm{NO}_{3}\right)_{3}$ standard solution $(1005 \mu \mathrm{g} / \mathrm{L}+5 \mathrm{wt} \%$ $\mathrm{HNO}_{3}$ ) and ruthenium(III) hexaammine trichloride were purchased from Aldrich. Sulfuric acid was from Carlo Erba reagents. All chemicals were used as received, and all aqueous solutions were prepared with deionised water purified via a Milli-Q unit (Millipore system). Unless otherwise stated, all measurements were performed in solutions purged with pure nitrogen $(99.99 \%$ from SIAD) and at room temperature $\left(21 \pm 1^{\circ} \mathrm{C}\right)$.

\subsection{Liquid Crystalline Plating Mixture}

The liquid crystalline plating mixture was composed of $42 \%(w / w) \mathrm{C}_{16} \mathrm{EO}_{8}, 29 \%(\mathrm{w} / \mathrm{w}) \mathrm{HCPA}$ and $29 \%(\mathrm{w} / \mathrm{w})$ Milli-Q water $[27,28,33,35]$. The normal topology $H_{I}$ phase was produced through efficient mixing of the components, employing a vortex mixer, while subjecting the mixture to three eating-cooling cycles from 20 to $80^{\circ} \mathrm{C}$ [33]. Prior to further use, the liquid crystalline phase was allowed to equilibrate for $24 \mathrm{~h}$ at room temperature.

\subsection{Instrumentation and Electrodes}

All electrochemical experiments were carried out in a two-electrode cell maintained in a Faraday cage made of sheets of aluminum to minimise external interference. Cyclic voltammetry $(\mathrm{CV})$ and chronoamperometry (CA) were performed by using the Potentiostat/Galvanostat $M$ 283 EG\&G PAR (Princeton, NY) in conjunction with the M 270 (EG \& G PAR) software. Unless otherwise stated, the reference electrode was an $\mathrm{Ag} / \mathrm{AgCl}$ saturated $\mathrm{KCl}$.

The smooth platinum disk microelectrodes were prepared by sealing $25 \mu \mathrm{m}$ diameter platinum wires into glass capillaries. All mesoporous platinum microelectrodes were prepared by electrodeposition of platinum films onto the platinum disk microelectrodes from the liquid crystalline plating mixture. The electrodeposition was carried out at constant potential at about $-0.6 \mathrm{~V}$ against a platinum spiral used as pseudo reference electrode. After deposition, the mesoporous electrodes were allowed to soak in Milli-Q water for $12-24 \mathrm{~h}$ to remove the surfactant.

The geometric radius of all microdisk electrodes (and consequently their geometric surface areas) was determined by recording the steady-state diffusion limiting current $\left(I_{\mathrm{L}}\right)$ from a $1 \mathrm{mM} \mathrm{Ru}\left(\mathrm{NH}_{3}\right)_{6} \mathrm{Cl}_{3}$ solution containing $0.1 \mathrm{M} \mathrm{KCl}$, and using the following equation [8].

$$
I_{\mathrm{L}}=4 n F D c^{\mathrm{b}} a
$$


where $n$ (equal to 1 ) is the number of electrons, $F$ is the Faraday constant, $D$ is the diffusion coefficient of the electroactive species $\left(7.0 \times 10^{-6} \mathrm{~cm}^{2} \mathrm{~s}^{-1}\right)[38], c^{\mathrm{b}}$ (equal to $1 \mathrm{mM})$ is the bulk concentration and $a$ is the radius of the microdisk. The steady state limiting current was determined by cyclic voltammetry at $5 \mathrm{mV} \mathrm{s}^{-1}$, where radial diffusion prevails $[8,10]$.

The real surface area of the various mesoporous $\mathrm{Pt}$ films was determined by cyclic voltammetry in $0.5 \mathrm{M}$ sulfuric acid $[20,33,39,40]$, and by analysis of the hydrogen underpotential deposition (H-UPD) process [39,40], which occurs over the potential region from -0.2 to $0.2 \mathrm{~V}$, vs. $\mathrm{Ag} / \mathrm{AgCl}$, and is due to the adsorption/desorption of a hydrogen monolayer onto the Pt surface. The charge involved in the H-UPD was converted to the real surface area using a conversion factor of $210 \mu \mathrm{Ccm}^{-2}$ $[40,41]$. The ratio between this surface and the geometric surface area of the electrode gave the roughness factor $(R F)$. In this work, MPtEs with $R F$ values in the range from about 2 (i.e., polished Pt microdisks) to 210 were investigated.

\subsection{Ex-situ Preparation of Bismuth Modified Mesoporous Platinum Microelectrodes (Bi-MPtEs)}

Bismuth modified mesoporous platinum microelectrodes were prepared by underpotential deposition of $\operatorname{Bi}(0)$ at a constant potential of $0.1 \mathrm{~V}[20,42]$, from a $1 \mathrm{mM}$ $\mathrm{Bi}\left(\mathrm{NO}_{3}\right)_{3}$ solution. The deposition time was varied from 1 to 3 min, depending on the $R F$ factor of the MPtE and on the bismuth coverage desired. The Bi-MPtEs thus prepared were taken out from the cell containing Bi(III) ions, rinsed with deionised water and then transferred, for further investigation, in a cell containing either $0.5 \mathrm{M}$ $\mathrm{H}_{2} \mathrm{SO}_{4}$ base electrolyte only or the base electrolyte and formic acid at different concentrations.

\section{Results and Discussion}

\subsection{Characterisation and stability of Bi-MPtEs}

Figure 1 compares typical CVs recorded with an MPtE in a $0.5 \mathrm{M} \mathrm{H}_{2} \mathrm{SO}_{4}$ solution before (full line) and after its modification with different amounts of adsorbed bismuth (dashed and dotted lines). Distinctive characteristics for the presence of bismuth ad-atoms on the platinum surface are the depression of the hydrogen adsorption/desorption peaks and the appearance of peaks I and I', which superimpose to those corresponding to Pt oxide formation and reduction. The depression of the hydrogen adsorption/desorption is due to the fact that hydrogen does not adsorb on $\operatorname{Bi}(0)$ [42], while the peaks $\mathrm{I}^{-\mathrm{I}^{\prime}}$ are due to the oxidation/reduction of $\mathrm{Bi}$ irreversibly adsorbed onto the $\mathrm{Pt}$ surface, the latter reactions occurring concomitantly to the metal oxide processes $[20,42]$. Since only the Pt sites unblocked from $\mathrm{Bi}$ are available for H-UPD, the fractional coverage of $\mathrm{Bi}$ ad-atoms $\left(\theta_{\mathrm{Bi}}\right)$ was evaluated from the charge involved in the hydrogen desorption before $\left(Q_{\mathrm{H}}{ }^{\mathrm{b}}\right)$ and after $\left(Q_{\mathrm{H}}{ }^{\mathrm{a}}\right)$ adsorption of $\mathrm{Bi}$ on the Pt electrode surface, by using the following relationship [20]:

$\theta_{\mathrm{Bi}}=\left(Q_{\mathrm{H}}{ }^{\mathrm{b}}-Q_{\mathrm{H}}{ }^{\mathrm{a}}\right) / Q_{\mathrm{H}}{ }^{\mathrm{b}}$

Bi-MPtEs with $\theta_{\mathrm{Bi}}$ values in the range $0-0.94$ were thus prepared. The stability of adsorbed Bi depended on the high potential limit set in the measurements. This was verified by performing a series of CVs starting from $-0.250 \mathrm{~V}$ and setting the upper potential limit at values where oxidised species of bismuth could be formed [43]. It was found that the initial amount of $\mathrm{Bi}$ on the Pt surface, i.e., that adsorbed from the $\mathrm{Bi}(\mathrm{III})$ containing solutions, was almost completely retained, provided that the upper potential limit was fixed below $0.55 \mathrm{~V}$. Higher potential limits above $0.55 \mathrm{~V}$ led to a progressive bismuth desorption upon continuous potential cycling, as was indicated by the increase of the hydrogen adsorption/desorption peaks and simultaneous decrease of the peak system I-I'. For instance, the CVs shown in Figure 1 with a dotted and dashed line were recorded with a Bi-MPtE $R F=65$ and $\theta_{\mathrm{Bi}}=0.70$ (initial value) over the potential window from -0.25 to $0.9 \mathrm{~V}$ after 20 and 30 cycles, respectively; correspondingly, the initial $\theta_{\mathrm{Bi}}$ value decreased to about 0.50 (dotted line) and 0.35 (dashed line).

\subsection{Formic Acid Oxidation}

Preliminarily, a series of cyclic voltammograms for the oxidation of formic acid was performed at unmodified MPtEs with different $R F$ factors, and Figure 2 shows typical CVs obtained for two MPtEs with $R F$ equal to 65 and 210 in $0.5 \mathrm{M} \mathrm{H}_{2} \mathrm{SO}_{4}$, containing $0.1 \mathrm{M} \mathrm{HCOOH}$. In these experiments the high potential limit was set at $0.9 \mathrm{~V}$. Under these conditions, two anodic peaks (1 and 2) in the direct scan, and the broad bell shaped wave (3), in the re-

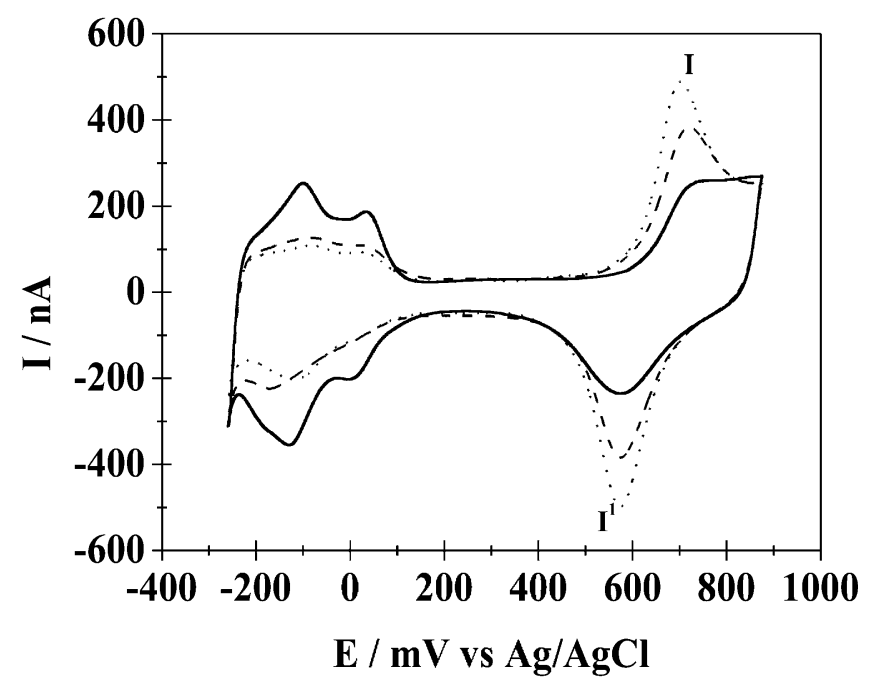

Fig. 1. Cyclic voltammograms recorded in a $0.5 \mathrm{M} \mathrm{H}_{2} \mathrm{SO}_{4}$ solution at a (-) MPtE $R F=65$ and at Bi-MPtE $R F=65$ and $\theta_{\mathrm{Bi}}=$ 0.70 after (---) $20 \mathrm{CV}$ cycles and after (.....) $30 \mathrm{CV}$ cycles. Potential window from -0.25 to $0.95 \mathrm{~V}$. Scan rate $50 \mathrm{mV} \mathrm{s}^{-1}$. 


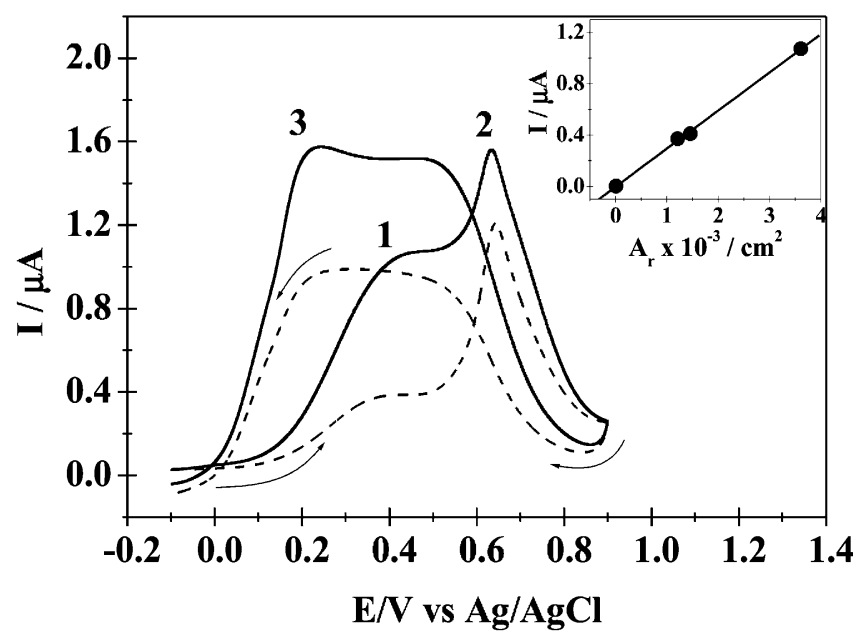

Fig. 2. Cyclic voltammograms obtained in a solution containing $0.5 \mathrm{M} \mathrm{H}_{2} \mathrm{SO}_{4}$ and $0.1 \mathrm{M} \mathrm{HCOOH}$ at an MPtE: $(-) R F=210$ and $(--) R F=65$. Scan rate $50 \mathrm{mV} \mathrm{s}^{-1}$. Inset: Plot of peak current (1) against real surface area $\left(A_{\mathrm{r}}\right)$.

verse scan were obtained. This voltammetric behaviour is in good agreement with those previously reported in the literature for smooth polycrystalline platinum electrodes [16,18,19,43-47], and reflects either the well-known dual paths mechanism $[46,48]$, or, as was recently proposed, the triple path mechanism [11,48]. That is, the direct formic oxidation to $\mathrm{CO}_{2}$ (dehydrogenation path), the route mediated by $\mathrm{CO}_{\text {ads }}$-like poison species (dehydration path), and the formate pathway, involving the formation and subsequent oxidation of adsorbed formate to $\mathrm{CO}_{2}$. Accordingly, the first forward peak (1) is attributed to the direct $\mathrm{HCOOH}$ oxidation occurring at surface sites that remain unblocked by $\mathrm{CO}$. The second forward peak (2) is related to the oxidation of adsorbed $\mathrm{CO}_{\text {ads }}$, which results from dehydration of formic acid. This electrode process leads to the release of the surface locations of the catalyst, and on the negative-going scan markedly larger anodic currents (wave 3) are observed, reflecting the $\mathrm{HCOOH}$ oxidation on the catalyst surface free from $\mathrm{CO}_{\text {ads }}$ [18]. From Figure 2 it is also evident that the increase of the real surface area of the MPtEs provides a general increase of the current over the entire potential window investigated, as expected for electrode processes occurring under kinetic control [26-28,30]. As for peak 1, its current increases almost linearly with real surface area as is evident from data included in inset of Figure 2.

The effect of $\mathrm{Bi}$ adsorbed on the Pt surface on the electrooxidation of $\mathrm{HCOOH}$ is shown in Figure 3, where a series of CVs performed at a range of Bi-MPtEs, having a fixed $R F$ and different $\theta_{\mathrm{Bi}}$ values, is displayed. As it is evident, by increasing bismuth coverage, the onset of the first forward wave shifts progressively towards less positive values (from $0.1 \mathrm{~V}$ at $\theta_{\mathrm{Bi}}=0$ down to $-0.1 \mathrm{~V}$ at $\theta_{\mathrm{Bi}}=$ 0.67 ), while its current increases up to four times greater than that of the uncoated MPtE. Peak 2 is somewhat lowered and is almost obscured for $\theta_{\mathrm{Bi}}>0.4$. The oxidation current of wave 3 in the reverse scan also increases as $\theta_{\mathrm{Bi}}$ increases, but to a smaller extent. These results suggest that, as the bismuth coverage increases, the formic acid dehydration rate becomes slower, and therefore smaller amounts of $\mathrm{CO}$ are eventually accumulated on the electrode surface during the direct scan. This $\mathrm{CO}$ removingeffect is very effective for $\theta_{\mathrm{Bi}}>0.3$, as also suggested by
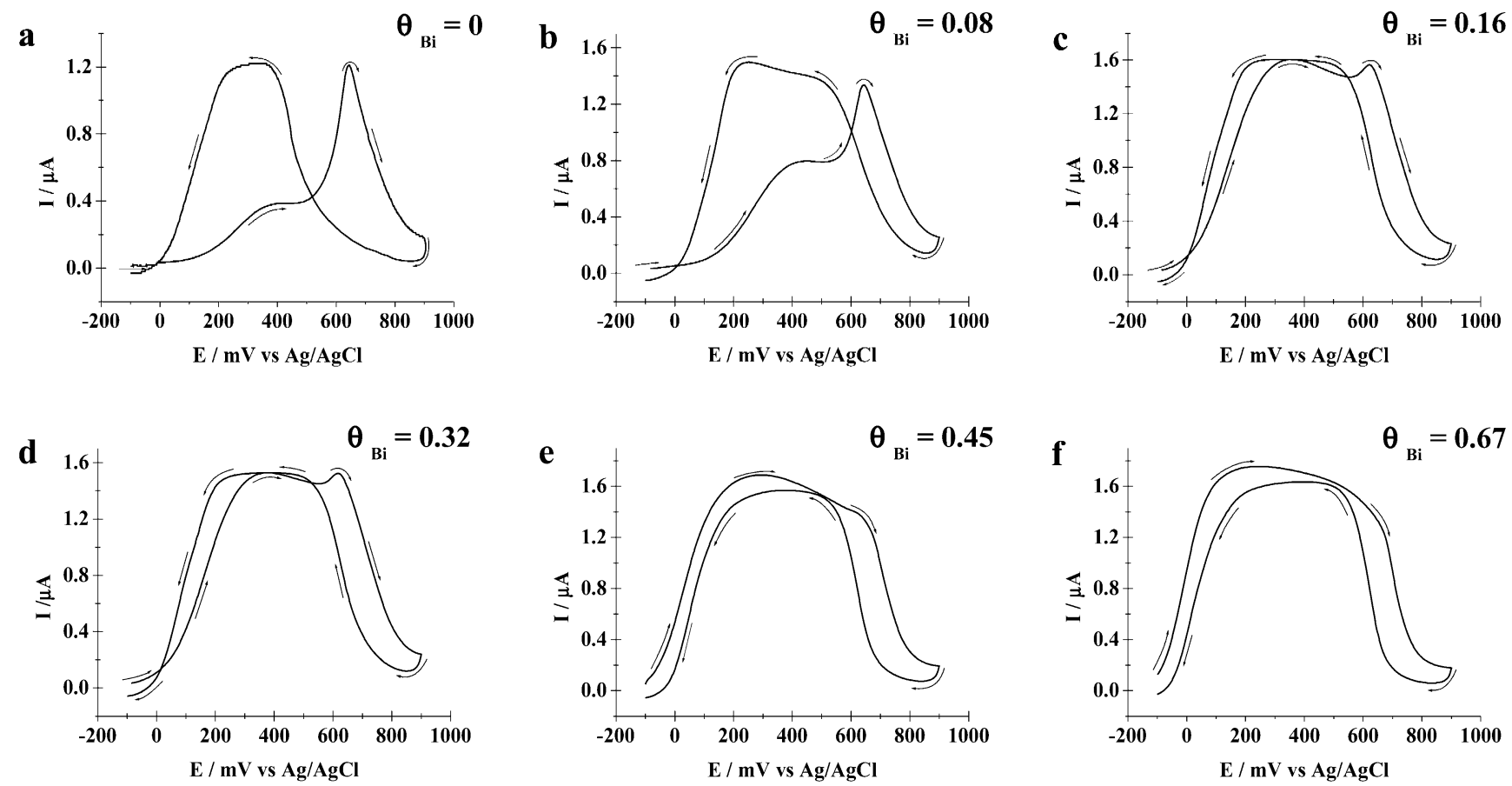

Fig. 3. Cyclic voltammograms obtained in a solution containing $0.5 \mathrm{M} \mathrm{H}_{2} \mathrm{SO}_{4}$ and $0.1 \mathrm{M} \mathrm{HCOOH}$ at an MPtE $R F=65$ modified by different amounts of irreversibly adsorbed bismuth, as indicated in the figure. Scan rate $50 \mathrm{mV} \mathrm{s}^{-1}$. 
the circumstance that, under the latter conditions, the currents for the forward scans were somewhat larger than those of the backward scans, while the maximum current shifts from $0.4 \mathrm{~V}$, at the MPtE, to $0.1 \mathrm{~V}$ at the Bi-MPtEs (Figure 3). It must be noticed that, the catalytic activity of the Bi-MPtEs observed here is congruent with those reported in the literature for polycrystalline platinum nanoparticles dispersed on various types of carbon supports, loaded by similar amounts of irreversibly adsorbed bismuth on the Pt surface $[5,22,23]$. As for the sudden decrease of current, which applies, regardless of the bismuth coverage, for potentials above $0.65 \mathrm{~V}$, it can conceivably be attributed to the formation of oxidised platinum and bismuth species on the electrode surface, which also have detrimental effects on the $\mathrm{HCOOH}$ electrooxidation process $[16,18,19,43-47]$. This can easily be inferred by comparing the voltammograms of Figures 1 and $3 \mathrm{e}-\mathrm{f}$.

As mentioned in the previous section, the high potential limits set in the cyclic voltammograms play an important role on the Bi-MPtEs stability. This in turn can affect the catalytic performance of the electrodes towards $\mathrm{HCOOH}$ electrooxidation upon continuous potential cycling. This aspect was investigated by performing $\mathrm{CV}$ measurements in which the high potential limit was set at values where Bi-UPD was either less or more stably adsorbed on to the electrode surface. Typical cyclic voltammograms recorded at Bi-MPtEs $R F=63$ and $\theta_{\mathrm{Bi}}=0.55$ in $0.1 \mathrm{M} \mathrm{HCOOH}$, upon continuous cycling the potential over the range from -0.1 to $0.9 \mathrm{~V}$ or -0.1 to $0.4 \mathrm{~V}$, are shown in Figure 4 and Figure $5 \mathrm{a}$, respectively. From Figure 4, it is evident that, in the case of higher potential limits, as the number of cycles increases, the features of the voltammogram progressively changes from that characteristic of high to that of low bismuth coverage (compare Figures 3 and 4). This is clearly due to the fact that adsorbed bismuth is progressively released from the electrode surface (see also section 3.1). On the other hand, when the high potential limit is set at $0.4 \mathrm{~V}$, rather stable waves with low hysteresis are recorded. Figure 5 a shows, as an example, the $1^{\text {st }}$ and $30^{\text {th }}$ cyclic voltammograms obtained under the latter conditions, which also testify the excellent reproducibility of the measurements. Furthermore, the shape of the CVs in Figure 5 a close resembles that expected for a microelectrode working under steadystate conditions, i.e., where a hemispherical diffusion regime prevails [10]. It must be remarked that investigations on $\mathrm{HCOOH}$ electrooxidation performed at a nanostructured palladium-gold alloy microelectrode also provided sigmoidal(S)-shaped waves [29]. Instead, no Sshaped voltammogram was ever recorded at bismuthmodified polycrystalline platinum electrodes of conventional size. These circumstances, therefore, suggest that mass transport play also a role in the electrooxidation process of $\mathrm{HCOOH}$ at the investigated Bi-MPtEs. The steady-state character of the current, which can be measured at potentials over the plateau region, was also checked by chronoamperometry. Figure $5 \mathrm{~b}$ shows typical current-time profiles obtained by setting $E=0.25 \mathrm{~V}$ and t equal to $30 \mathrm{~s}$ or $180 \mathrm{~s}$. As it is evident, the current decreases rapidly at short times and then reaches an almost constant value, which, at $30 \mathrm{~s}$, corresponds, within $5 \%$, to that obtained by $\mathrm{CV}$ at the same potential.

Measurements as those described above were also performed by using Bi-MPtEs with either a fixed $R F$ or dif-
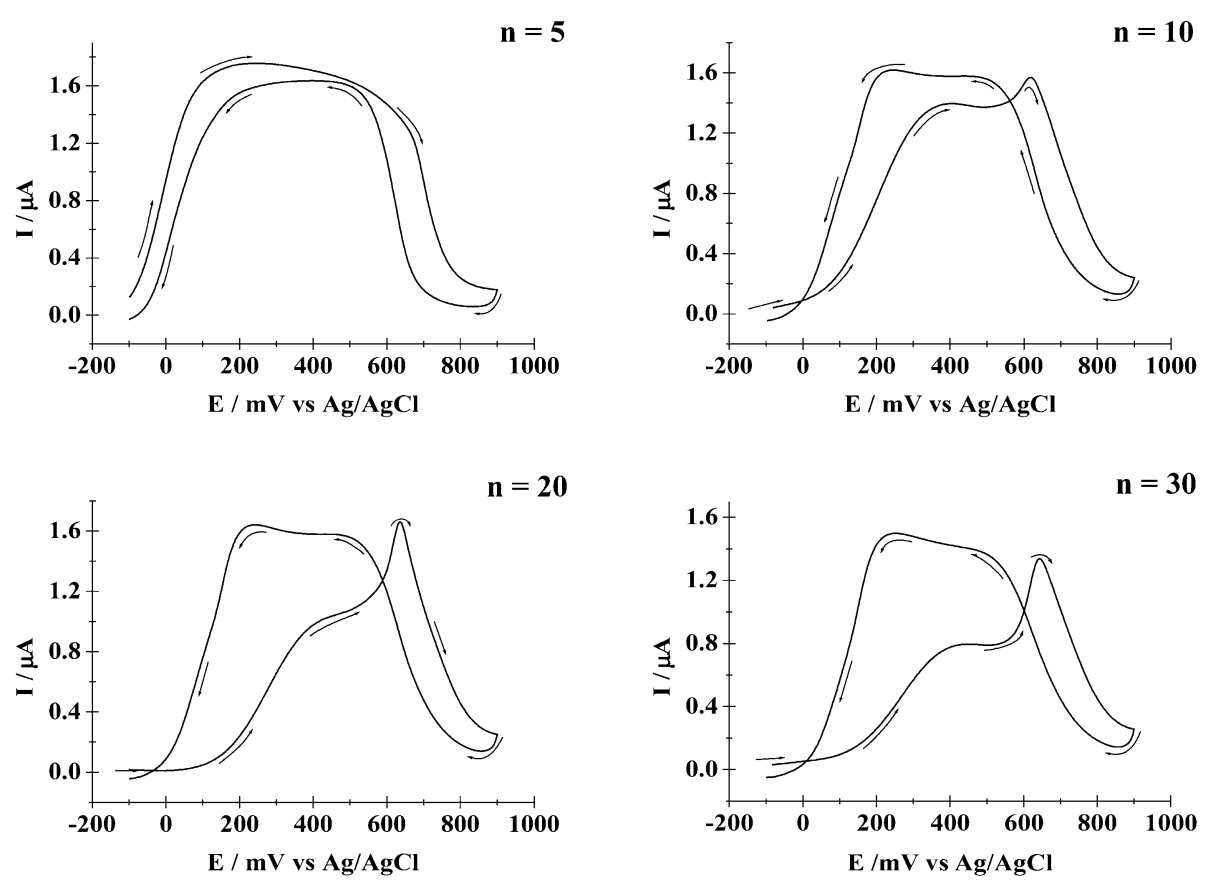

Fig. 4. Cyclic voltammograms obtained in a solution containing $0.5 \mathrm{M} \mathrm{H}_{2} \mathrm{SO}_{4}$ and $0.1 \mathrm{M} \mathrm{HCOOH}$ at a $\mathrm{Bi}-\mathrm{MPtE} R F=65$ and $\theta_{\mathrm{Bi}}=$ 0.55 . Effect of cycling over the potential window from -0.10 to $0.95 \mathrm{~V}$. Number $(n)$ of cycles indicated in the figure. Scan rate $50 \mathrm{mV} \mathrm{s}^{-1}$. 

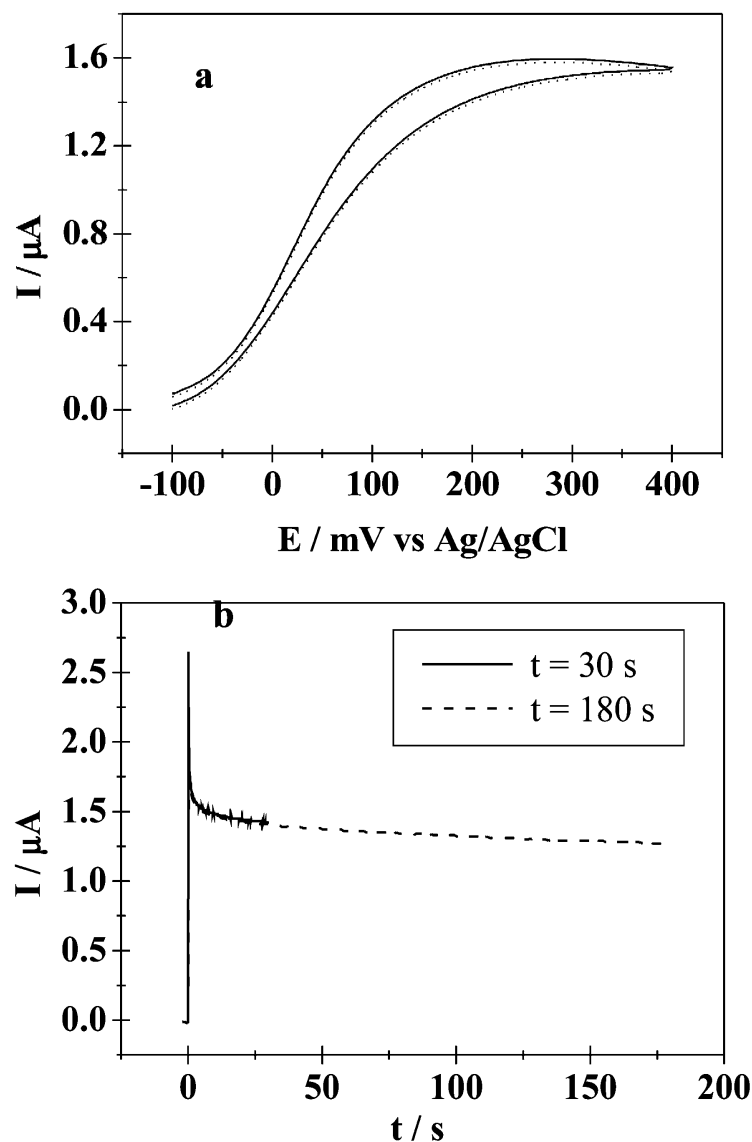

Fig. 5. (a) Cyclic voltammograms obtained in a solution containing $0.5 \mathrm{M} \mathrm{H}_{2} \mathrm{SO}_{4}$ and $0.1 \mathrm{M} \mathrm{HCOOH}$ at a Bi-MPtE $R F=65$ and $\theta_{\mathrm{Bi}}=0.55$. Effect of cycling over the potential window from -0.1 to $0.4 \mathrm{~V} ;(-) 1^{\text {st }}$ cycle, $(---) 30^{\text {th }}$ cycle. Scan rate $50 \mathrm{mV} \mathrm{s}^{-1}$. (b) Chronoamperometry performed at $0.25 \mathrm{~V}$ for $30 \mathrm{~s}$ and $180 \mathrm{~s}$ in the same solution as above.

ferent initial $\theta_{\mathrm{Bi}}$ values, and vice versa. Apart from the specific currents involved, in general, behaviours as those described previously were found. However, comparison of the current values normalised for the real surface area indicated that current density was somewhat larger as the $\mathrm{RF}$ of the Bi-MPtEs increased. This suggested that real surface area and bismuth coverage could have a cooperative effect on the $\mathrm{HCOOH}$ oxidation process.

The effect of bulk concentration of the $\mathrm{HCOOH}$ on the activity of two Bi-MPtEs was investigated over the range $10 \mathrm{mM}-5 \mathrm{M}$ by using cyclic voltammetry and chronoamperometry. In order to preserve the stability of bismuth adlayers, in these measurements, the high potential limit was set at $0.4 \mathrm{~V}$. Bi-MPtEs with a fixed $R F=210$ and $\theta_{\mathrm{Bi}}$ equal to 0.6 and 0.26 were examined. Comparative measurements were also performed with the corresponding unmodified MPtE over the same concentration range. Figure 6 shows a series of CVs obtained at the $\mathrm{Bi}$ MPtE $R F=210$ and $\theta_{\mathrm{Bi}}=0.6$ in $\mathrm{HCOOH}$ solutions at different concentrations, while Figure 7 displays current against concentration plots obtained over the entire concentration range examined for the two Bi-MPtEs and the MPtE investigated. In the latter case, the current of pro-

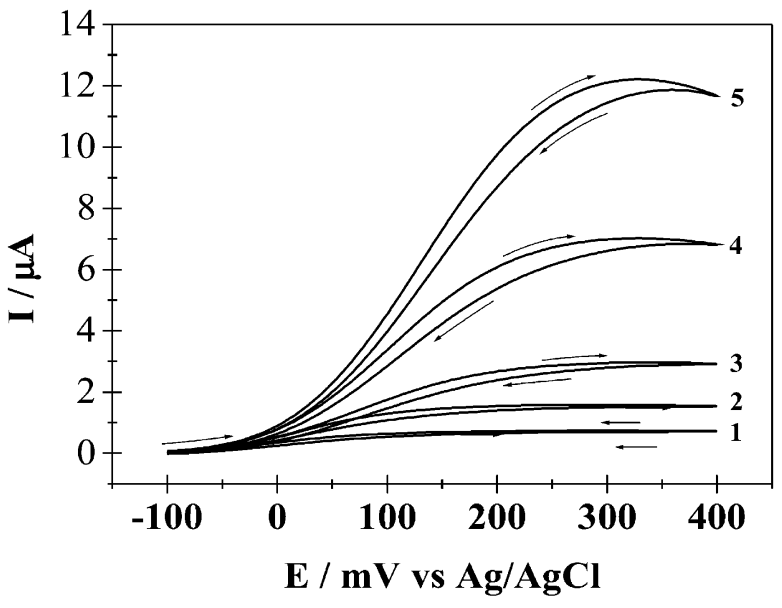

Fig. 6. Cyclic voltammograms recorded at a Bi-MPtE $R F=210$ and $\theta_{\mathrm{Bi}}=0.6$ in $0.5 \mathrm{M} \mathrm{H}_{2} \mathrm{SO}_{4}$ containing $\mathrm{HCOOH}$ (1) $0.05 \mathrm{M}$, (2) $0.1 \mathrm{M}$, (3) $0.02 \mathrm{M}$, (4) $0.5 \mathrm{M}$, (5) $1 \mathrm{M}$. Scan rate $50 \mathrm{mV} \mathrm{s}^{-1}$.

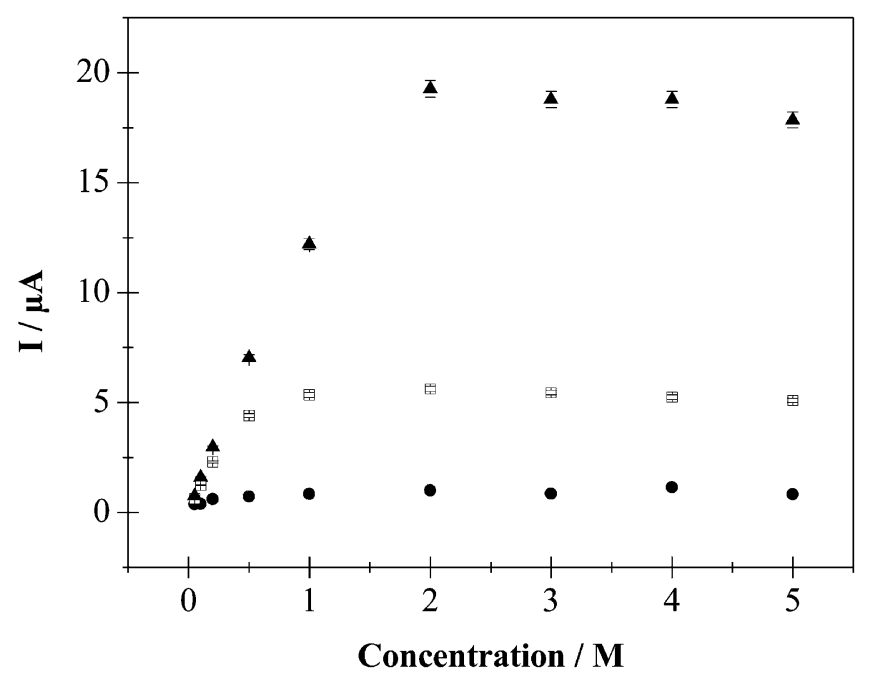

Fig. 7. Calibration plots for $\mathrm{HCOOH}$ obtained from $\mathrm{CV}$ in a solution containing $0.5 \mathrm{M} \mathrm{H}_{2} \mathrm{SO}_{4}$ at Bi-MPtEs with $R F=210$ and $\theta_{\mathrm{Bi}}$ equal to $(\bullet) 0,(\square) 0.25,(\mathbf{\Delta}) 0.6$.

cess (1) in Figure 1 was considered for the construction of the plots. Calibration plots (not shown) as those displayed in Figure 7 were also obtained by taking the steady-state currents from chronoamperometry (at $30 \mathrm{~s}$ or $120 \mathrm{~s}$ ). In all cases, the current increased up to a value, which was the higher the larger the bismuth coverage, after which the current levelled off or even decreased (Figure 7). From linear regression analysis of experimental data, linear dynamic ranges and slopes for the various electrodes were evaluated (Table 1). All calibration parameters were higher at the Bi-PtME with $\theta_{\mathrm{Bi}}=0.6$, at which current increased linearly up to $2 \mathrm{M}$. The decrease of current at high $\mathrm{HCOOH}$ concentrations suggests that the oxidation rate of $\mathrm{HCOOH}$ also decreases. This negative effect, as expected, is more dramatic for Bi-PtMEs with lower bismuth coverage and for the MPtEs. Reproducibility and long term stability of the Bi-PtMEs were investigated by 
Table 1. Regression parameters of current against $\mathrm{HCOOH}$ concentration plots obtained at a Bi-PtME $R F=210$ with different amounts of adsorbed bismuth.

\begin{tabular}{llll}
\hline$\theta_{\mathrm{Bi}}$ & Dynamic range $(\mathrm{M})$ & Slope $(\mu \mathrm{A} / \mathrm{M})$ & $R^{2}$ \\
\hline 0 & $0.01-0.1$ & $1.70 \pm 0.03$ & 0.978 \\
0.25 & $0.01-0.5$ & $10.9 \pm 0.1$ & 0.990 \\
0.60 & $0.01-2.0$ & $13.8 \pm 0.1$ & 0.995 \\
\hline
\end{tabular}

running repetitive measurements over the concentration range, where linearity applied. It was found that reproducibility was within $2 \%(R S D$.) (at least four replicates were performed at each concentration) provided that the sensors were employed under the above optimised experimental conditions of upper potential limits. Long term stability was also ascertained by using the Bi-PtME $R F=$ 210 and $\theta_{\mathrm{Bi}}=0.6$. Measurements were performed by running 2-3 CVs each day for three consecutive months. It was found that the investigated electrode system essentially kept its initial performance (within $0.3-0.4 \% /$ week) over a period of two months. Afterwards, the current values, at a given $\mathrm{HCOOH}$ concentration, progressively decreased $(\sim 1 \% /$ week $)$, probably for the partially damage of the mesoporous nanostructure. The loss of adsorbed $\mathrm{Bi}$, which also could deteriorate the sensor performance, could be easily recovered by reloading the electrode surface with the appropriate amount of bismuth.

\section{Conclusions}

In this paper it has been shown that mesoporous platinum microelectrodes modified with even small amounts of adsorbed bismuth enhances the formic acid oxidation current. However, the stability of the Bi-MPtEs has been found to depend on higher potentials set in the experiments. In particular, potentials greater than $0.5 \mathrm{Vvs}$. Ag/ $\mathrm{AgCl}$ resulted in the progressive release of $\mathrm{Bi}$ from the $\mathrm{Pt}$ surface, and consequently in the decrease of the catalytic activity towards the electrooxidation of $\mathrm{HCOOH}$. Good performance has instead been found when the operation potentials were set at values $\leq 0.4 \mathrm{~V}$. Bi-MPtEs with relatively high $\mathrm{Bi}$ coverage (i.e., $\bar{\theta}_{\mathrm{Bi}} \geq 0.6$ ) have displayed sigmoidal voltammograms as those expected for microelectrodes working under a steady state. It has also been verified that the steady state current is proportional to the bulk concentration of $\mathrm{HCOOH}$ over a concentration range that depended on the $\mathrm{Bi}$ loading the mesoporous platinum. Bi-MPtEs and $\theta_{\mathrm{Bi}} \geq 0.6$ provided a linear dynamic range up to $2 \mathrm{M}$ with high repeatability. These analytical features are suitable for monitoring, and thus control, the crossover of HCOOH in DFAFC [49].

\section{Acknowledgements}

Financial support of the Ministry of University and Scientific Research (MUR, PRIN 2008 MWHCP2) is gratefully acknowledged.

\section{References}

[1] V. P. McConnell, Fuel Cells Bull. 2009, 6, 12.

[2] X. Yu, P. G. Pickup, J. Power Sources 2008, 182, 124.

[3] J. Lipkowski, P. N. Ross, Electrocatalysis, Wiley-VCH, New York 1998

[4] J. M. Feliu, E. Herrero, in Handbook of Fuel Cells. Fundamental and Applications (Ed: W. Vielstich, A. Lamm, H. A. Gasteiger), Wiley, New York 2003.

[5] W. Qian, D. P. Wilkinson, J. Shen, H. Wang, J. Zhang, J. Power Sources 2006, 154, 202.

[6] S. Uhn, H. J. Lee, Y. Kwon, J. Lee, Angew. Chem. Int. Ed. 2008, 47, 10163.

[7] C. Rice, S. Ha, R. I. Masel, A. Wieckowski, J. Power Sources 2003, 115, 229.

[8] A. J. Bard, L. R. Faulkner, Electrochemical Methods, Wiley, New York 2001.

[9] K. T. Jeng, W. M. Huang, C. C. Chien, N. Y. Hsu, Sens. Actuators $B$ 2007, 125, 278.

[10] M. I. Montenegro, M. A. Queiros, J. L. Daschbach, Microelectrodes: Theory and Applications, NATO ASI Series, Vol. E197 1991.

[11] Y. X. Chen, M. Heinen, Z. Jusys, R. J. Behm, Angew. Chem. Int. Ed. 2006, 45, 981.

[12] S. E. Habas, H. Lee, V. Radmilovic, G. A. Somorjai, P. Yang, Nat. Mater 2007, 6, 692.

[13] Y. X. Chen, S. Ye, M. Heinen, Z. Jusys, M. Osawa, R. J. Behm, J. Phys. Chem. B 2006, 110, 9534.

[14] J. Jiang, A. Kucernak, J. Electroanal. Chem. 2002, 520, 64.

[15] W. Zhou, Y. Du, H. Zhang, J. Xu, P. Yang, Electrochim. Acta 2010, 55, 2911.

[16] S. Kang, J. Lee, J. K. Lee, S. Y. Chung, Y. Tak, J. Phys. Chem. B 2006, 110, 7270 .

[17] J. Jiang, A. Kucernak, Chem. Mater. 2004, 16, 1362.

[18] G. Q. Lu, A. Crown, A. Wieckowski J. Phys. Chem. B 1999, 103, 9700 .

[19] A. V. Tripković, K. Dj. Popovic, R. M. Stevanovic, R. Socha, A. Kowal Electrochem. Commun. 2006, 8, 1492.

[20] S. Daniele, S. Bergamin, Electrochem. Commun 2007, 9, 1388.

[21] J. Jiang, A. Kucernak, J. Electroanal. Chem. 2009, 630, 10.

[22] B. J. Kim, K. Kwon, C. K. Rhee, J. Han, T. H. Lim, Electrochim. Acta 2008, 53, 7744.

[23] A. Lopez-Cudero, F. J. Vidal Iglesias, J. Solla-Gullon, E. Herrero, A. Aldaz, J. M. Feliu, Phys. Chem. Chem. Phys. 2009, 11, 416.

[24] M. D. Obradovic, A. V. Tripkovic, S. L. Gojkovic, Electrochim. Acta 2009, 55, 204.

[25] J. Jiang, A. Kucernak, J. Electroanal. Chem. 2002, 533, 153.

[26] A. Kucernak, J. Jiang, Chem. Eng. J. 2003, 93, 81.

[27] P. Birkin, J. M. Elliot, Y. E. Watson, Chem. Commun. 2000, 17, 1693.

[28] S. A. G. Evans, J. M. Elliot, L. M. Andrews, P. N. Bartlett, P. J. Doyle, G. Denuault, Anal. Chem. 2002, 74, 1322.

[29] J. Jiang, A. Kucernak, Electrochim. Acta 2009, 54, 4545.

[30] S. Daniele, D. Battistel, S. Bergamin, C. Bragato, Electroanalysis 2010, 22, 1511.

[31] G. Denuault, Isr. J. Chem. 2010, 50, 374.

[32] G. S. Attard, P. N. Bartlett, N. R. B. Coleman, J. M. Ellliott, J. R. Owen, J. H. Wang, Science 1997, 278, 838.

[33] J. M. Elliott, P. R. Birkin, P. N. Bartlett, G. S. Attard, Langmuir 1999, 15, 7411.

[34] P. A. Nelson, J. M. Elliott, G. S. Attard, J. R. Owen, Chem. Mater. 2002, 14, 524.

[35] P. Lozano Sanchez, J. M. Elliott, Analyst 2005, 130, 715.

[36] M. A. Baldo, S. Daniele, Electroanalysis 2006, 18, 633. 
[37] R. L. C. Thiago Paixao, L. Juliana. L. Cardoso, M. Bertotti. Fuel 2007, 86, 1185.

[38] D. Battistel, S. Daniele, G. Battaglin, M. A. Baldo, Electrochem. Commun. 2009, 11, 2195.

[39] S. Trasatti, O. A. Petrii, Pure Appl. Chem. 1991, 63, 711.

[40] M. Noel, K. I. Vasu. Cyclic Voltammetry and the Frontiers of Electrochemistry, Aspect Publications, London, 1990.

[41] T. Biegler, D. A. J. Rand, R. Woods, J. Electroanal. Chem. 1971, 29, 269.

[42] S. H. Cadle, S. Bruckenstein, Anal. Chem. 1972, 44, 1993.

[43] S. Uhm, Y. Yun, Y. Tak, J. Lee, Electrochem. Commun. 2005, 7, 1375.
[44] E. Casado-Rivera, D. J. Volpe, L. Alden, C. Lind, C. Downie, T. Vazquez-Alvarez, A. C. D. Angelo, F. J. Di Salvo, H. D. Abruna, J. Am. Chem. Soc. 2004, 126, 4043.

[45] Z. H. Wang, K. Y. Qu, Electrochem. Commun. 2006, 8, 1075.

[46] A. Capon, R. Parsons J. Electroanal. Chem. 1973, 44, 239.

[47] A. Wieckowski, J. Sobkowski J. Electroanal. Chem. 1975, $63,365$.

[48] Y. X. Chen, S. Ye, M. M. Heinen, Z. Jusys, M. Osawa, R. J. Behn J. Phys. Chem. B. 2006, 110, 9534.

[49] Y. W. Rhee, S. Y. Ha, R. I. Masel, J. Power Sources, 2003, 117,30 . 